\title{
The distance to the Large Magellanic Cloud from first overtone RR Lyrae variables
}

\author{
A.V. Muzzin, C.M. Clement \\ Department of Astronomy \& Astrophysics, University of Toronto, 60 St. \\ George St., Toronto, Ontario, M5S 3H8, Canada \\ D.R. Alves \\ Columbia Astrophysics Laboratory, 550 W. 120th St., Mailcode 5247, \\ New York, New York, 10027, USA \\ \& The MACHO Collaboration
}

\begin{abstract}
Fourier coefficients have been derived for the $V$ and $R$ light curves of 330 bona fide RR Lyrae first-overtone (RR1) pulsators in 16 MACHO fields near the bar of the LMC. We use the Fourier phase parameter $\phi_{31}$ and $\log P$ values to select a subsample of these stars which are similar to the RR1s in the Galactic globular cluster M5. Assuming that the M5-like stars in the LMC have absolute magnitudes comparable to the $\mathrm{HB}$ stars in M5, we use independent studies to derive their mean absolute $V$ magnitude and compute a visual distance modulus of $\mu_{L M C}=18.43 \pm 0.06$ (statistical) \pm 0.16 (systematic). By selecting stars on the basis of their light curve parameters, we are able to derive a distance modulus that does not depend on the somewhat poorly determined $M_{v}-[\mathrm{Fe} / \mathrm{H}]$ relation for RR Lyr stars.
\end{abstract}

\section{Introduction}

The determination of the LMC distance modulus $\left(\mu_{L M C}\right)$ has had a long, and somewhat controversial history. The controversy arose because typically Population II distances indicators such as RR Lyr stars, gave short values of the distance modulus $(\leq 18.40$ ), while Population I indicators, such as Cepheids, gave long values of $\mu_{L M C}(\geq 18.60)$. Contributing to the problem is the fact that a single measurement of the distance modulus is fraught with many problems: 1) It is difficult to be sure that the population of standard candles used for the distance determination is homogeneous. 2) Stars in the cloud suffer from significant differential reddening. 3) The fields near the LMC are highly crowded and therefore absolute photometry is extremely challenging. 4) The cloud itself is inclined to the line-of-sight and one must allow for this. Our measurement of the LMC distance modulus carefully addresses all of these issues. 

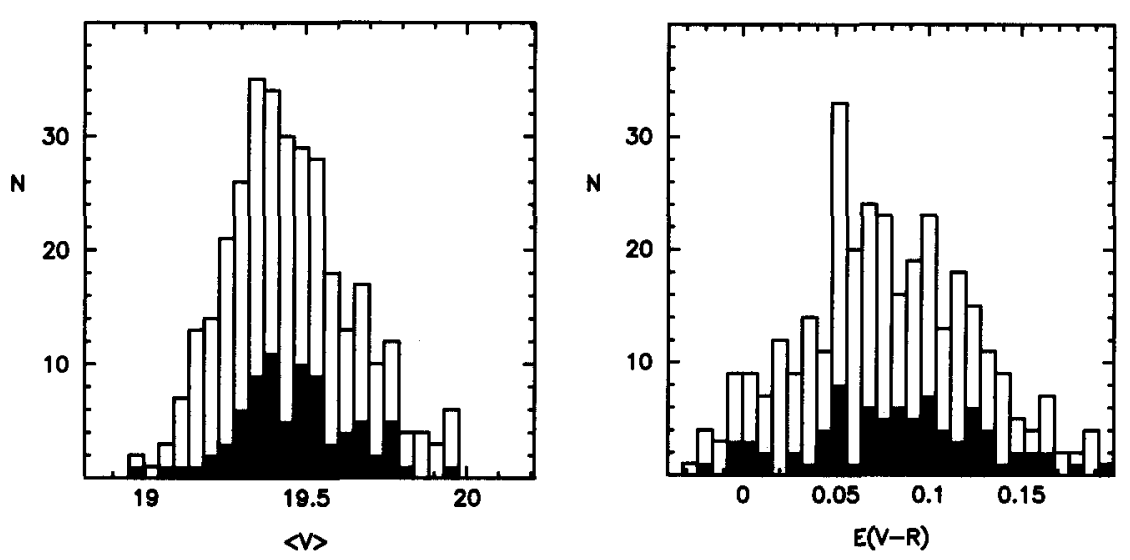

Figure 1. Histograms of mean apparent $V$ magnitude and extinction for the 330 bona fide RR1 variables. The filled histogram represents M5-like stars.

\section{The selection of a homogeneous sample}

Previous investigations by Simon \& Clement (1993), Kovács \& Jurcsik (1996), and Kovács (1998), have shown that Fourier coefficients derived from the light curves of RR Lyr variables (particularly the phase parameter $\phi_{31}$ ) are related to the luminosity of the stars. Therefore, stars with similar luminosities can be selected using their Fourier parameters. To select such a group of stars, we use the sample of 330 bona fide RR1 stars identified by Alcock et al. 2004 (hereafter, A04) in the MACHO project variable star database.

Based on a plot of $\phi_{31}$ vs. $\log$ P, A04 compared the LMC RR1 stars to those found in some well-studied Galactic globular clusters (GGCs). They found that of the 330 LMC RR1 variables there were many that had properties similar to the variables in both M5 and M3. They also found that the RR1 variables in the Galactic GCs M68, M107, NGC 6441, and the Oosterhoff type II population of $\omega$ Centauri had few counterparts amongst the LMC stars.

M3 has more RR Lyr variables than M5 and there are probably more M3like stars in the LMC. However, we selected the M5-like variables because the $\phi_{31}-\log \mathrm{P}$ relation determined by Kaluzny et al. (2000) for M5 is better than any available for M3. Our sample of M5-like LMC stars consists of 80 stars.

\section{Interstellar reddening}

The severe differential reddening that occurs in stars in the LMC makes it optimal to compute dereddened apparent magnitudes for stars on a one by one basis, if possible. To do this, we employ equations derived by Simon \& Clement (1993) to calculate $\log T_{\text {eff }}$ in terms of $\phi_{31}$ and $\log P$. We then convert these temperatures to unreddened colors using the color-temperature relation of Kovács \& Walker (1999). Comparing the expected color of the RR1 variables with the observed colors allows us to compute the $V-R$ color excess, $\mathrm{E}(V-R)$. 

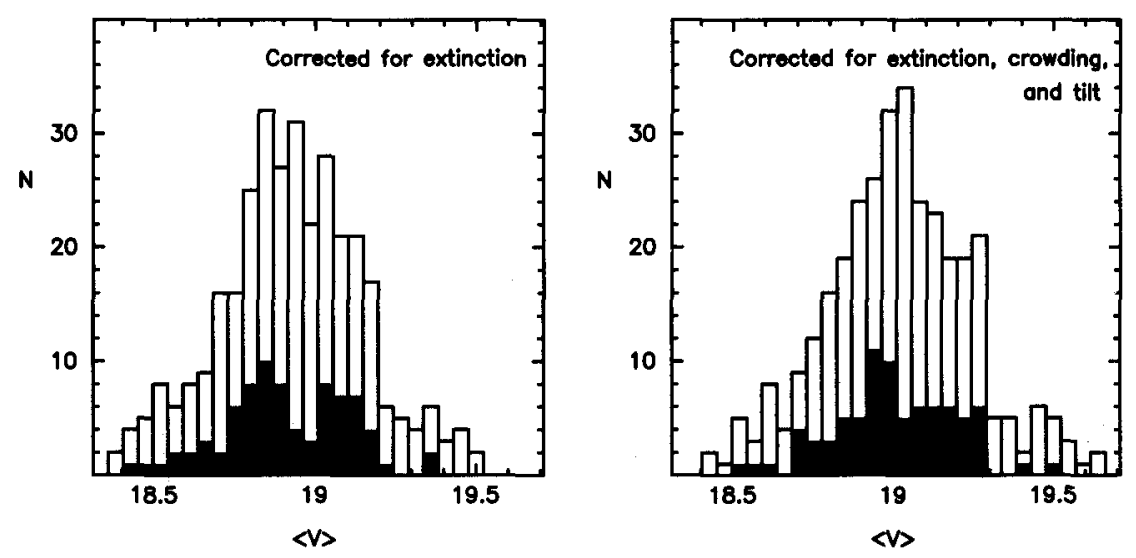

Figure 2. Histograms of $\langle V\rangle$ for all $330 \mathrm{RR} 1$ stars with reddening correction only (panel 1), and with reddening, crowding, and depth corrections (panel 2). Again, filled histograms represent the M5-like stars. The typical systematic error in $\langle V\rangle$ due to reddening alone is \pm 0.12 .

The second panel of Fig. 1 shows a histogram of $\mathrm{E}(V-R)$ for all stars (lined) and M5-like stars (filled). For the sample of 330 bona fide RR1 stars we find $\langle\mathrm{E}(V-R)\rangle=0.08 \pm 0.003$ and for the M5-like stars $\langle\mathrm{E}(V-R)\rangle=0.09 \pm 0.005$, where the errors quoted are the statistical errors. The typical systematic error in a single star's reddening is \pm 0.02 , due to model uncertainties. Applying the reddening corrections to our sample of $330 \mathrm{RR} 1 \mathrm{~s}$ results in the mean dereddened $\langle V\rangle$ being $18.91 \pm 0.01$ and for the M5-like stars it is $18.90 \pm 0.02$, the errors again being statistical. The first panel of Fig. 2 presents the histogram for these dereddened $\langle V\rangle$ values.

\section{The crowding and tilt corrections}

Artificial star tests performed by the MACHO project (Alcock et al. 2001) showed that in order to recover the true luminosity of stars in the dense region near the LMC bar, crowding corrections are required. We performed additional artificial star tests in 10 areas of the LMC which spanned the range of crowding levels in our 16 fields in order to determine the appropriate corrections. The tests showed that the mean increase in brightness due to crowding effects ranged between 0.05 and 0.15 mag.

Using equations derived by van der Marel \& Cioni (2001), we also applied a correction for the tilt of the cloud. The second panel of Fig. 2 shows a histogram of the final dereddened, crowding corrected, and tilt corrected values of $\langle V\rangle$. Once all corrections have been made, it is clear that the bimodal distribution of M5-like stars which appears in the left panel of Fig. 2 is no longer evident. The fully corrected mean $\left\langle V_{0}\right\rangle$ for the M5-like stars that we adopt for the distance determination is $18.99 \pm 0.02$. 


\section{The LMC distance modulus}

To determine the LMC distance we apply absolute magnitudes for RR Lyr stars in M5 computed from several independent methods: Baade-Wesselink analyses (Clementini et al. 1995), main sequence fitting (Gratton et al. 2003), pulsation models (Simon \& Clement 1993), and the trigonometric parallax of RR Lyr (Benedict et al. 2002). A weighted average of these measurements gives $\mathrm{M}_{v}=0.56 \pm 0.06$. Combining this with our $\left\langle V_{0}\right\rangle$ gives $\mu_{\mathrm{LMC}}=18.43 \pm 0.06$ (statistical) \pm 0.16 (systematic). The main source of the systematic error is from the uncertainty in the crowding correction, as well as the uncertainty in the stellar models used for deriving the reddening. A more detailed presentation of the methods and results of this study can be found in A04.

\section{References}

Alcock, C., et al. 2001, ApJS, 136, 439

Alcock, C., et al. 2004, AJ, 127, in press (A04)

Benedict, G.F., et al. 2002, AJ, 123, 473

Clementini, et al. 1995, AJ, 110, 2319

Gratton, R.G., et al. 2003, A\&A, 408, 529

Kaluzny, J., et al. 2000, A\&AS, 143, 215

Kovács, G. 1998, Mem. Soc. Astron. Italiana, 69, 49

Kovács, G., Jurcsik, J. 1996, ApJ, 466, L17

Kovács, G., Walker, A.R. 1999, ApJ, 512, 271

Simon, N.R., Clement, C.M. 1993, ApJ, 410, 526

van der Marel, R.P., Cioni, M.L., 2001, AJ, 122, 1807 